\title{
Comparative Study on Serodiagnosis of Bovine Leptospirosis by Microagglutination Test (MAT) and Indirect ELISA
}

\section{Blessy Rajan, Sumanth Kumar, Raghavan Madhusoodanan Pillai, Prabhakar Xavier Antony, Hirak Kumar Mukhopadhyay, Sangeetha Balakrishnan, Nobal Rajkumar and Mouttou Vivek Srinivas*}

Department of Veterinary Microbiology, Rajiv Gandhi Institute of Veterinary Education and Research, Pondicherry-605 009, India

*Corresponding author

\section{A B S T R A C T}

\section{Keywords}

Bovine leptospirosis, Indirect ELISA, Outer membrane protein (OMP),

Microagglutination test (MAT).

\section{Article Info}

Accepted:

24 January 2017

Available Online:

10 February 2017
The present study was undertaken to identify the presence of anti-leptospiral antibodies in the bovine serum samples using two serological tests, a genus specific indirect ELISA and a serovar specific microagglutination test (MAT). A total of 250 serum samples from bovine leptospirosis suspected cases were sampled in and around Pondicherry (Southern India). The anit-leptospiral antibodies were detected by indirect ELISA using outer membrane proteins (OMP's) of Leptospira interrogans serovar pomona as antigen and compared with MAT using six infecting serovars (hardjo, icterohaemorrhagiae, grippotyphosa, pomona, canicola and australis) as antigen. Statistical analysis of the data was performed using chi-square test. Out of 250 sera samples, 91 (36.4\%) and $62(24.8 \%)$ were positive by indirect ELISA and MAT respectively. The indirect ELISA employed in this study revealed a very high sensitivity of $98.38 \%$ in comparison to $67.03 \%$ in MAT. Chi -square analysis revealed a significant difference (i.e. $\mathrm{P}<0.001$ ) between the two serological tests. This study revealed that indirect ELISA was found to be highly sensitive, rapid and easy to perform for detection of bovine anti-leptospiral antibodies in comparison with MAT.

\section{Introduction}

Leptospirosis is one of the most important zoonotic diseases with worldwide distribution and public health concern. In domestic animals like cattle, it is an important cause of abortion, stillbirth, infertility, decreased milk production and death (Bharti et al., 2003).

This zoonotic disease is caused by the genus Leptospira which comprises of more than 260 serovars classified under 24 serogroups based on agglutinating antigens and is further classified into multiple genomospecies based on their genetic variations (Cerqueira et al., 2009).

The laboratory diagnosis of the disease is based on either isolation, identification of leptospires or the detection of anti-leptospiral antibodies. The isolation, identification of leptospires is time-consuming and necessitates specialized reference laboratories. The microscopic examination of leptospires by dark field microscopy contributes to the early diagnosis of the 
infection. However it has two major drawbacks, i) too low concentration of leptospires (less than $10^{4}$ cells $/ \mathrm{ml}$ ) may not be detected and ii) presence of artefacts such as fibrin and extrusions from cellular elements can be easily mistaken for Leptospira by inexperienced workers (Faine, 1982).

The serological tests have now become the corner stone for diagnosis of leptospirosis. The universally accepted test, Microagglutination test (MAT) is hazardous to perform, as live Leptospira culture is used as antigen and the test is serovar specific. Moreover it is tedious and time consuming (Subharat et al., 2012; de Oliveirai et al., 2013; Khalili et al., 2014). Leptospiral outer membrane proteins (OMP) contain transmembrane proteins like porin (OMP L1) and lipoproteins. Because of antigenic variability, leptospiral Lipopolysaccharide (LPS) has a limitation for serodiagnosis. Ideally an antigen of choice is one which is highly conserved among the pathogenic leptospiral serovars. In this context OMPs show good potential as they are conserved within the various pathogenic serovars. Such outer membrane proteins and associated proteins selectively solubilised using Triton-X 114 detergent will be of immense diagnostic value (Bolin et al., 1989).

Enzyme linked immunosorbent has been found to be a more sensitive serological test than the conventional methods for diagnosis of leptospirosis (Sumanth et al., 2013; Ye et al., 2015). ELISA is one of the most commonly used type of screening test because of its relatively simple methodology, inherent high sensitivity, and suitability for testing large numbers of test samples. This test has been used for a wide range of infectious diseases and also to detect leptospira specific antibodies in humans and animals (Senthilkumar et al., 2010; Sumanth et al.,
2013). Hence the present study was aimed for detection of bovine anti-leptospiral antibodies using serological test like indirect ELISA and compare it with internationally standard serovar specific microagglutination test (MAT) for its specificity and sensitivity.

\section{Materials and Methods}

\section{Ethical approval}

The approval from the Institutional Animal Ethics Committee (IAEC) to carry out this study was not required as no invasive technique was used. Serum samples were being collected from bovine leptospirosis suspected cases from Veterinary hospitals and Veterinary Dispensaries in Pondicherry.

Bacterial cultures: Eight standard serovars of Leptospira viz; Leptospira interrogans serovars hardjo, canicola, icterohaemorrhagiae, pomona, grippotyphosa, australis, autumnalis and hebdomadis were obtained from the National Leptospira Laboratory, Port Blair, Andaman and Nicobar Islands and were maintained at the Department of Veterinary Microbiology, Rajiv Gandhi Institute of Veterinary Education and Research, Pondicherry.

Serum samples: A total of 250 serum samples from bovine leptospirosis suspected cases were sampled in and around Pondicherry (Southern India). No vaccination program against bovine leptospirosis has been carried out in these regions.

Anti sera: Hyper immune serum was raised against $L$. interrogans serovar pomona in calves by repeated injection of formalinkilled antigen. Serum dilution ranging from $1: 10$ to $1: 100$ dilution of hyperimmune sera raised against Leptospira interrogans serovar pomona was used. 
OMPs Extraction: The outer membrane proteins from Leptospira interrogans serovar pomona was extracted by triton X-114 method as described by Haake et al., (1999). By this method, the leptospires were washed in PBS-5 $\mathrm{mM} \mathrm{MgCl} 2$ and then extracted in the presence of $1 \%$ protein grade Triton $\mathrm{X}$ $114-150 \mathrm{mM} \mathrm{NaCl}-10$ mMTris (pH 8)-1 $\mathrm{mM}$ EDTA at $4{ }^{\circ} \mathrm{C}$. The insoluble material was removed by centrifugation at $17,000 \times \mathrm{x}$ for $10 \mathrm{~min}$. After centrifugation, $20 \mathrm{mM}$ $\mathrm{CaCl}_{2}$ was added to the supernatant. Phase separation was performed by warming the supernatant to $37^{\circ} \mathrm{C}$ and subjecting it to centrifugation for $10 \mathrm{~min}$ at $1,000 \mathrm{x} \mathrm{g}$. The detergent and aqueous phases were separated and precipitated with acetone.

Optimization of OMP antigen: An end point titration was carried out to determine the optimum single working dilution of OMP antigen by serial two fold dilution (using carbonate -bicarbonate buffer $\mathrm{pH}-9.6$ ) of antigen so as to provide dilution range from $6.1 \mu \mathrm{g} /$ well to $0.09 \mu \mathrm{g} /$ well across each column of ELISA plate with each column contain varying dilution of hyperimmune serum $(1: 10$ to $1: 100)$.

Indirect ELISA: Flat bottomed 96 well polystyrene microtiter plates were coated with antigen in carbonate - bicarbonate buffer and incubated overnight at $4^{\circ} \mathrm{C}$. The plates were washed five times with PBS (pH 7.2) containing $0.05 \%$ Tween 20 (PBST). Plates were incubated with blocking solution (5\% skim milk powder) for $1 \mathrm{hr}$ at $37^{\circ} \mathrm{C}$. The plates were washed as above and $100 \mu$ of 1:40 diluted test serum samples was added to individual wells in duplicates followed by incubation at $37^{\circ} \mathrm{C}$ for one hour. The plates were washed and $100 \mu l$ of 1:1000 dilutions of rabbit anti-bovine HRPO conjugate (DAKO, Agilent) was added to all the wells and again incubated at $37^{\circ} \mathrm{C}$ for one hour. After washing with PBST, $100 \mu$ l of freshly prepared OPD - $\mathrm{H}_{2} \mathrm{O}_{2}$ substrate solution was added to each well, and the plate was kept at room temperature $\left(25^{\circ} \mathrm{C}\right)$ for $10 \mathrm{~min}$ at dark. The enzyme substrate reaction was stopped by adding $50 \mu \mathrm{l}$ of $1 \mathrm{M} \mathrm{H}_{2} \mathrm{SO}_{4}$. The optical density of the samples was recorded at 492 nm by ELISA reader (Tecan Infinite 50).

Microagglutination test (MAT): Antibodies to leptospira were detected by MAT according to Faine [3]. The panel of reference L. interrogans serovars included in the study were hardjo, canicola, icterohaemorrhagiae, pomona, grippotyphosa and australis. A serum dilution of 1:50 was first used to screen the samples for leptospirosis. The positive samples were identified and titrated using serial two fold dilutions from 1:50 to 1:3200 in the following manner. $100 \mu \mathrm{l}$ of PBS was added to wells 2 to 8 in 96 well flat bottomed microtitre plate. Then $100 \mu \mathrm{l}$ of pre-diluted serum (1:25) was added to wells 1 and 2 including positive controls corresponding to those serovars. Doubling dilution of sera was made from well 2 to 8 . $100 \mu$ l of antigen (5-7 day old live culture) was added to wells 1 to 8 so that the final dilution was $1: 50$ to $1: 6400$. The mixture was gently tapped to mix the contents and incubated at $37^{\circ} \mathrm{C}$ for 2 to 4 hours. A drop of the mixture was taken on a microscopic slide and observed under dark field microscope for agglutination.

Evaluation of MAT and indirect ELISA: The relative sensitivity and specificity of indirect ELISA were evaluated in comparison to MAT as described by Thrushfield (1995).

Statistical analysis: Relative sensitivity and specificity of indirect ELISA were determined in comparison with MAT, as described by Zweing and Robertson (1987). Comparative evaluations of both serological tests were carried out by chi-square test (SPSS 17.0 software). 


\section{Results and Discussion}

\section{Antigen extraction and Protein estimation:}

Seven day old culture of Leptospira interrogans serovar pomona was used to obtain OMPs by Triton X-114 extraction method. This method was found to be satisfactory as bulk amount of antigen could be obtained. The concentration of outer membrane protein extracted was $4.094 \mu \mathrm{g} / \mu \mathrm{l}$ determined by Lowry's method. The OMP antigen based diagnostic test has advantages over MAT like safety because latter requires the use of live organisms and also it can able detects genus specific antibodies.

\section{Standardization of Indirect ELISA:} Indirect ELISA was standardized by optimising antigen dilution at varying dilutions of hyperimmune sera ranging from $1: 10$ to $1: 100$ and serum from confirmed healthy calves as negative control and rabbit anti-bovine IgG-HRPO conjugate at a constant dilution of 1:1000. The OD values were recorded for the varying dilutions of OMP antigen against a constant dilution of hyperimmune serum in each column (Figure2). It was observed that an antigen concentration of $0.768 \mu \mathrm{g} / \mathrm{well}$ at $1: 40$ dilution of hyperimmune sera and 1:1000 dilution of rabbit anti-bovine IgG-HRPO was found to be satisfactory for screening the test samples. (Table-1). At an antigen concentration of $0.768 \mu \mathrm{g}$, the OD value of strong positive was 1.022. Ribotta et al. (2000) used a much higher concentration of antigen, $2.2 \mu \mathrm{g}$ per well. But in the present study a minimal concentration of antigen was used, likely due to the quality and purity of the antigen extracted. Previously Sumanth et al., (2013) has used Leptospira interrogans serovar canicola for OMP antigen extraction for the development of ELISA for the detection of canine anti-leptospiral antibodies in this same laboratory. In their study, the OMP concentration of $0.4575 \mu \mathrm{g} /$ well was found to be satisfactory to screen the test serum samples for canine anti-leptospiral antibodies. So in this study, an antigen concentration of $0.768 \mu \mathrm{g} / \mathrm{well}$ was taken as optimal antigen concentration to coat the nunc maxisorb ELISA plates for screening the test serum samples for bovine anti-leptospiral antibodies.

Determination of ELISA cut-off value and calculation of percent positivity (PP) values: A concentration of $0.768 \mu \mathrm{g}$ per well of antigen, average OD values of 1.23 and 0.21 were obtained for strong positive and healthy serum respectively. The PP values were calculated using the average OD values of negative control serum samples. Based on these OD values, the mean PP value of the negative control group was calculated as 17.07. Therefore, any serum sample showing a PP value of 34.14 (Twice the mean negative $\mathrm{PP}$ value) or more was considered as positive for leptospirosis. Out of the 250 serum samples, $91(36.4 \%)$ were positive for leptospirosis by indirect ELISA. The conversion of raw OD values into PP values offers advantage, as it rules out the variations arising out of OD value of test samples in comparison with control values of each plate on day to day basis. Thus the results interpreted based on PP values offer means to distinctly separate a population of animals which are positive and negative.

Microagglutination test: The titre of the highest dilution at which 50\% agglutination was observed (Figure-1). Out of the 250 bovine serum samples tested against 6 serovars of Leptospira, $62(24.8 \%)$ serum samples were found to be positive. In that 26 $(41.94 \%)$ serum samples were found to be positive for serovar hardjo, 15 (24.19\%) for serovar grippotyphosa, 10 (16.13\%) for serovar pomona, 7 (11.29\%) for serovar icterohaemorrhagiae, $4(6.45 \%)$ for serovar canicola and no serum sample was found to 
be positive for serovar australis (Figure-3). It was observed that 26 serum samples cross reacted with various serovars. A total of 8 serum samples cross reacted with grippotyphosa and canicola; 7 sera with hardjo and icterohaemorrhagiae; 6 sera with hardjo and pomona; 3 sera with grippotyphosa and icterohaemorrhagiae and 2 with grippotyphosa and pomona. These cross reacted sera were checked in different dilutions to find out the serovar.

Of the many important members of pathogenic serovars of Leptospira interrogans, six serovars were selected in this study to test the antibodies in the bovine sera. Several MAT-based studies have revealed various reactive serovars in cattle and serovar hardjo has been reported as the most important throughout the world (Garoussi et al., 2015). L. interrogans serovars pomona, icterohaemorrhagiae and grippotyphosa are non-host adapted for cattle and sporadically infect cattle as accidental hosts, causing acute disease and abortion (Miller et al., 1991). In addition, serovars like icterohaemorrhagiae, hardjo, patoc, australis, caniocola, grippotyphosa, pyrogenes, pomona, tarassovi and ballum were also reported responsible for sero-positivity among most of the animals and humans (Srivastava and Kumar, 2003). According to this study the frequency of leptospiral infection with hardjo serovar is more common among cattle in Pondicherry (Southern India).

Sensitivity and specificity of indirect ELISA and MAT: The sensitivity and specificity of the indirect ELISA in comparison with MAT was $98.38 \%$ and $84.04 \%$ respectively (Table-2). But the sensitivity and specificity of MAT in comparison to indirect ELISA was $67.03 \%$ and $99.37 \%$ respectively. The indirect ELISA developed by Ribotta et al., (2000) showed higher sensitivity (100\%) and higher specificity $(95.6 \%)$ than the present study. Rana et al., (2012) has shown sensitivity and specificity $100 \%$ and $92.92 \%$ respectively using rLipL32 as a coating antigen.

The relative sensitivity of indirect ELISA obtained in our study was $98.38 \%$ suggesting that it was as effective as MAT in detecting true positive animals. However, the specificity was $84.04 \%$ signifying the inability of the ELISA to detect all the MAT negative animals as ELISA negative too. This could be explained on the basis that all those sera turned out to be MAT negative may not actually be negative as only 6 Leptospira serovars were used as antigens for antibody detection in MAT. However, the findings supported the prevailing view that the ELISA was more sensitive than MAT (Rana et al., 2012; Deneke et al., 2014).

Statistical analysis: Chi-square analysis of the data obtained in this study for ELISA and MAT tests revealed that there is a significant difference between indirect ELISA and MAT employed in this study (where chi-square value is 25.2903 with Probability value 0.0001 i.e., <0.01) so, it is highly significant at $1 \%$ level.

Table.1 End point titration to find out the optimum concentration of OMP (coating) antigen for indirect ELISA

\begin{tabular}{lrrrrrrr}
\hline Hyperimmune serum & \multicolumn{7}{c}{ 1:40 dilution } \\
\hline OMP antigen concentration $(\mu \mathrm{g} / \mu \mathrm{l})$ & 6.14 & 3.07 & 1.53 & 0.76 & 0.38 & 0.19 & 0.09 \\
OD value $(492 \mathrm{~nm})$ & 1.155 & 1.143 & 1.043 & 1.022 & 0.896 & 0.682 & 0.537 \\
\hline
\end{tabular}


Table.2 Relative sensitivity and specificity of MAT compared with indirect ELISA for the detection of anti-leptospiral antibodies in bovine sera

\begin{tabular}{lrrr}
\hline \multirow{2}{*}{ ELISA } & \multicolumn{3}{c}{ MAT } \\
\cline { 2 - 4 } & Positive & Negative & Total \\
\hline Positive & 61 & 30 & 91 \\
Negative & 1 & 158 & 159 \\
Total & 62 & 188 & 250 \\
\hline
\end{tabular}

Fig.1 Photomicrograph of Microagglutination Test (MAT) using dark-field microscopy (a) Live leptospiral organism with no agglutination (Negative control), (b) 50\% Agglutination, (c) 100\% Agglutination
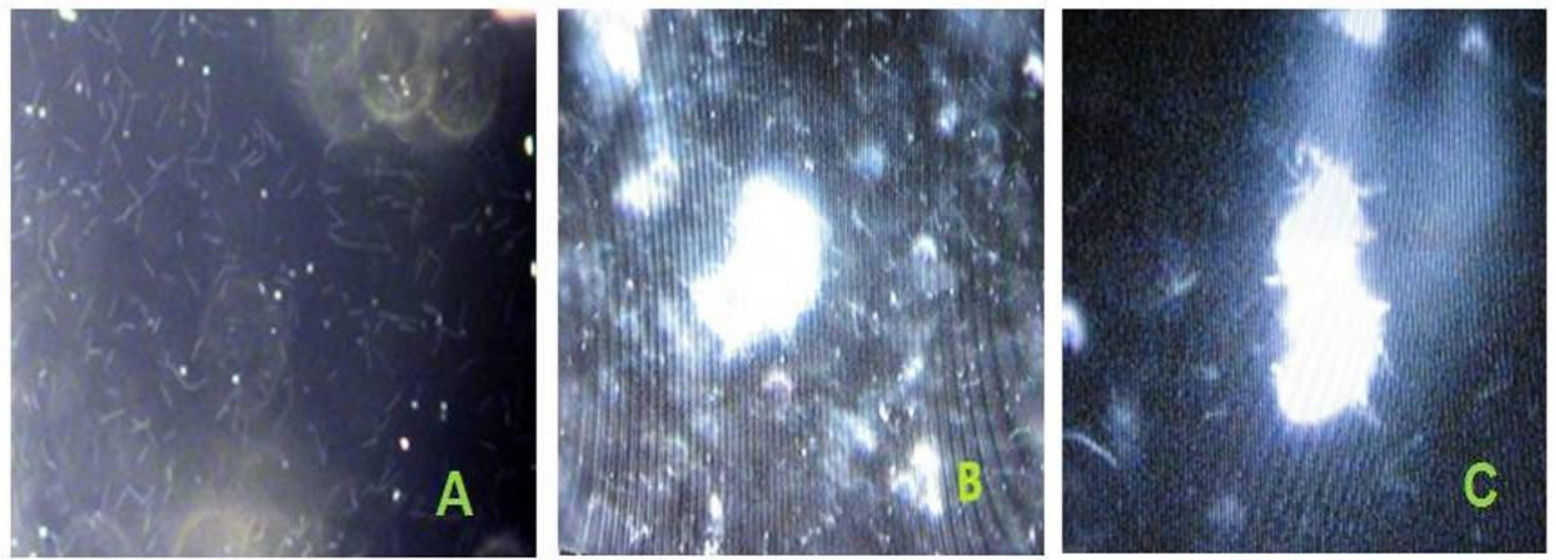

Fig.2 Determination of optimum concentration of OMP (coating) antigen for indirect ELISA

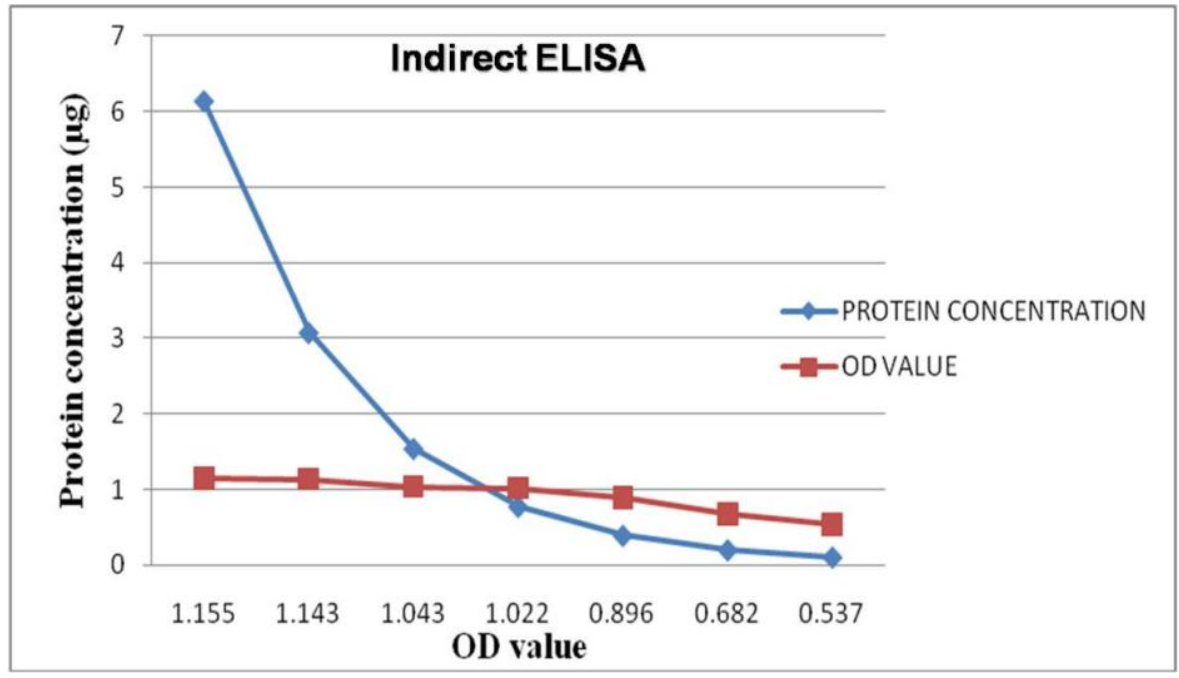


Fig.3 Comparative seroprevalence of leptospiral serovars in the bovine population by Microagglutination Test (MAT)

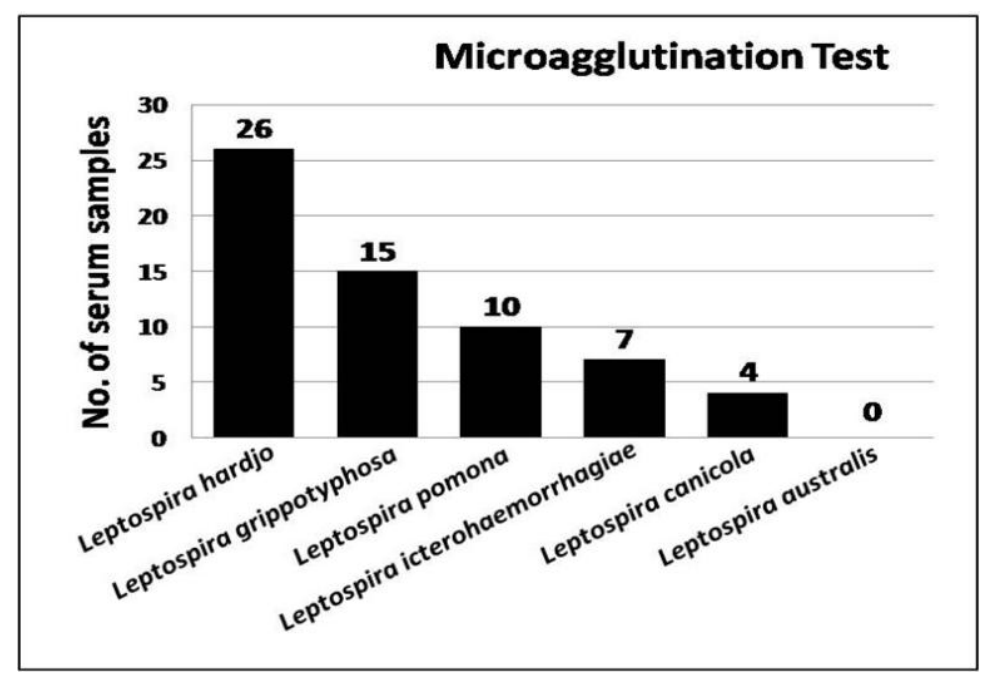

The present study concluded that the indirect ELISA was found to be highly sensitive, rapid and easy to perform in comparison with microagglutination test for the detection of bovine anti-leptospiral antibodies. By MAT it was found that the predominant serovar causing leptospirosis in cattle in this study was Leptospira interrogans serovar hardjo. In comparison to MAT, the Indirect ELISA employs a non-hazardous, highly purified and reproducible antigenic preparation which can be prepared in large quantity and could be stored for long periods.

In addition to this, the assay does not require the maintenance of live leptospiral cultures of different serovars as in the case of MAT. Moreover, ELISA could be objectively interpreted, and also has repeatability in the test. Therefore indirect ELISA can be recommended as a valuable test to perform for detection of bovine anti-leptospiral antibodies in comparison with MAT.

\section{References}

Bharti, A.R., Nally, J.E., Ricaldi, J.N., Matthias, M.A., Diaz, M.M., Lovett, M.A., Levett, P.N., Gilman, R.H., Willig, M.R.,
Gotuzzo, E.and Vinetz, J.M. 2003. Leptospirosis: a zoonotic disease of global importance, Lancet Infect.Dis., 3(12): 757771.

Bolin, C.A., Zuerner, R.L. and Trueba, G. 1989. Comparison of three techniques to detect Leptospira interrogans serovar hardjo type hardjo-bovis in bovine urine, Am.J. Vet.Res., 50:1001-1003.

Cerqueira, G.M.and Picardeau, M. 2009. A century of Leptospira strain typing, Infect. Genet. Evol., 9(5): 760-768.

Deneke, Y., Sabarinath, T., Gogia, N., Lalsiamthara, J., Viswas, K.N. and Chaudhuri, P. 2014. Evaluation of recombinant LigB antigen-based indirect ELISA and latex agglutination test for the serodiagnosis of bovine leptospirosis in India, Mol.Cell Probes, 28(4):141-6.

Faine, S. 1982. Guidelines for the the control of leptospirosis, World Health Organization, Geneva.

Garoussi, M.T., Mehravaran, M., Abdollahpour, G.and Khoshnegah, J. 2015. Seroprevalence of leptospiral infection in feline population in urban and dairy cattle herds in Mashhad, Iran, Vet.Res.Forum, 6(4):301-4.

Haake, D.A. et al., 1999. Leptospiral outer membrane proteins OmpL1 and lipL41 exhibit synergistic immunoprotection. 
Infect. Immun., 67: 6572-6582. Khalili, M., Sakhaee, E., Aflatoonian, M.R., Abdollahpour, G., Tabrizi, S.S., Damaneh, E.M. and Hossini-nasab, S. 2014. Seroprevalence of bovine leptospiral antibodies by microscopic agglutination test in Southeast of Iran, Asian Pac.J.Trop.Biomed., 4(5):354-357.

Miller, D.A., Wilson, M.A., and Beran, G.A. 1991. Survey to estimate prevalence of Leptospirainterrogans infection in mature cattle in the United States, Am.J.Vet.Res., 52:1761-1765.

de OliveiraI, R.M., Silva, M.L.C.R., MacêdoI, M.M.S., HiginoI, S.S.S., PaulinII, L.M., AlvesI, C.J., de CarvalhoI, M.G.X.and de AzevedoI, S.S. 2013. Seroepidemiology of bovine leptospirosis and brucellosis in family farm rural properties in the State of Paraíba, northeastern Brazil, Arq.Inst.Biol., 80:3.

Rana, A.S., Sinha, D.K., Chaudhury, P., Atre, H.S., Singh, V.P. and Srivastava, S.K. 2012. Comparative study on seroepidemiology of bovine leptospirosis by microagglutination test (MAT) and recombinant lip132 ELISA, J.Vet.Public Health, 10(2): 91-96.

Ribotta, M.J., Higgins, R., Gottschalk, M.and Lallier, R. 2000. Development of an Indirect enzyme linked immunosorbent assay for the detection of leptospiral antibodies in dogs, Can.J.Vet.Res., 64:3237.

Senthikumar, T.M.A., Subathra, M., Ramadass, P. and Ramaswamy, V. 2010. Serodiagnosis of bovine leptospirosis by IgG- Enzyme-linked Immunosorbent

\section{How to cite this article:}

Blessy Rajan, Sumanth Kumar, Raghavan Madhusoodanan Pillai, Prabhakar Xavier Antony, Hirak Kumar Mukhopadhyay, Sangeetha Balakrishnan, Nobal Rajkumar and Mouttou Vivek Srinivas. 2017. Comparative Study on Serodiagnosis of Bovine Leptospirosis by Microagglutination Test (MAT) and Indirect ELISA. Int.J.Curr.Microbiol.App.Sci. 6(2): 15511558. doi: http://dx.doi.org/10.20546/ijcmas.2017.602.173
Assay and Latex agglutination test, Trop.Anim.Health Prod., 42:217-222.

Srivastava, S.K., and Kumar, A.A. 2003. Seroprevalence of leptospirosis in animals and human beings in various regions of the country, Ind. J. Comp. Microbiol. Immunol.Infect.Dis., 24(2):155-15.

Subharat, S., Wilson, P., Heuer, C.and CollinsEmerson, J. 2012. Longitudinal serological survey and herd-level risk factors for Leptospira spp.serovars Hardjo-bovis and Pomona on deer farms with sheep and/or beef cattle, $N$ Z.Vet.J., 60(4):215-22.

Sumanth Kumar, R., Pillai, R.M., Mukhopadhyay, H.K., Antony, P.X., Thanislass, J., Vivek Srinivas, V.M.and Vishnupriya, S. 2013. Seroepidemiology of canine leptospirosis by iELISA and MAT, Vet.World, 6(11):926-930.

Thrusfield, M. 1995. Veterinary Epidemiology, $2^{\text {nd }}$ ed.The Blackwell Science publications, U.K.

Ye, C., Yan, W., McDonough, P.L., McDonough, S.P., Mohamed, H., Divers, T.J., Chang, Y. and Yang, Z. 2015. Serodiagnosis of Equine Leptospirosis by Enzyme-Linked Immunosorbent Assay Using Four Recombinant Protein Markers, Clin.Vaccine Immunol., 21(4):478-483.

Zweing, M.H. and Robertson, E.A. 1987. Clinical validation of immunoassays: a welldesigned approach to a clinical study, In: Chaw DW, Perlstein MT, eds.Immunoassay: A Practical Guide, San Diego: Academic Pr.97-127. 